\title{
IIIEST:Clêncla
}

\section{ESTIMATIVA DO BALANÇO HÍDRICO CLIMATOLÓGICO E CLASSIFICAÇÃO CLIMÁTICÁ PARA O MUNICÍPIO DE BOM JESUS DA LAPA-BA}

\author{
ESTIMATE OF CLIMATOLOGICAL WATER BALANCE AND CLIMATE \\ CLASSIFICATION FOR THE MUNICIPALITY OF BOM JESUS DA LAPA-BA
}

\author{
Taiara Souza Costa ${ }^{1} *$ \\ Ramon Amaro de Sales ${ }^{2}$ \\ Robson de Jesus Santos ${ }^{3}$ \\ Robson Argolo dos Santos ${ }^{1}$ \\ Rosangela Leal Santos ${ }^{3}$
}

\begin{abstract}
${ }^{1}$ Departamento de Engenharia Agrícola -Universidade Federal de Viçosa. E-mail: taiarasouzacosta1@ gmail.com.
${ }^{2}$ Departamento de Fitotecnia, Universidade Federal de Viçosa. E-mail: ramonamarodesales@gmail.com

${ }^{3}$ Universidade Estadual de Feira de Santana. E-mail: robssantos17@hotmail.com

${ }^{4}$ Departamento de Engenharia Agrícola -Universidade Federal de Viçosa. E-mail: argolo.agro@gmail.com

${ }^{5}$ Universidade Estadual de Feira de Santana. E-mail: rosangela.leal@gmail.com. *e-mail para correspondência: taiarasouzacosta1@gmail.com
\end{abstract}

Artigo submetido em 27/06/2019, aceito em 06/09/2019 e publicado em 20/12/2019.

\begin{abstract}
Resumo: O objetivo deste trabalho foi analisar a disponibilidade hídrica através do Balanço Hídrico Climatológico e realizar a Classificação Climática, pelo método de Thornthwaite para o município de Bom Jesus da Lapa, Bahia. Para elaboração do Balanço Hídrico Climatológico foram utilizados dados mensais de precipitação e temperatura sendo esses dados provenientes de uma série de 38 anos, pertencente ao Instituto Nacional de Meteorologia. Foi adotado o valor de $100 \mathrm{~mm}$ para a capacidade de água disponível no solo (CAD). A Classificação Climática utiliza os dados de excesso e deficiência hídrica anual obtida do Balanço Hídrico Climatológico. Os resultados obtidos mostraram que a temperatura média anual foi de $26,37^{\circ} \mathrm{C}$ e a precipitação média anual é de $792,66 \mathrm{~mm}$. Os meses de Janeiro a Novembro compreendem o período de deficiência hídrica, enquanto que Dezembro é representado por reposição hídrica. Quanto a Classificação Climática, o município possui o clima $\mathrm{C}_{1}$ $\mathrm{w}_{2} \mathrm{~A}^{\prime}$ a' que corresponde ao clima sub-úmido seco com grande deficiência hídrica no inverno, megatérmico com concentração de evapotranspiração potencial no verão igual a 36,64\% em relação à evapotranspiração anual.
\end{abstract}

Palavras-chave: Semiárido; déficit hídrico; solo; planta.

Abstract: The objective of this study was to analyze the water availability using the Climatological Water Balance and to perform the Climate Classification by the Thornthwaite method for the municipality of Bom Jesus da Lapa, Bahia. For the elaboration of the Climatological Water Balance, monthly data of temperature and temperature were used, being this data of a series of 38 years, 
belonging to the National Institute of Meteorology. The value of $100 \mathrm{~mm}$ was adopted for the available groundwater capacity (CAD). The Climate Classification uses the data of excess and annual water changes that are used in the Climatic Water Balance. The results show that the average annual temperature was $26.37{ }^{\circ} \mathrm{C}$ and the annual average was $792.66 \mathrm{~mm}$. The months from January to November comprise the period of water deficiency, while December is represented by water replacement. Regarding the climate classification, the municipality has a climate $\mathrm{C}_{1} \mathrm{w}_{2}$ A' a' Which corresponds to a sub-humid climate, with great water deficiency in winter, megathermic with potential evapotranspiration concentration in summer equal to $36.64 \%$ in relation to annual evapotranspiration.

Keywords: Semi-arid; water deficit; ground; plant

\section{INTRODUÇÃO}

Em conformidade ao cenário de aumento populacional e consequentemente elevação da demanda alimentar, é visível a preocupação da humanidade nos últimos tempos, em relação à disponibilidade de água em diversos cenários nacionais e internacionais (SALES et al., 2018). Com destaque para a região Nordeste brasileira, a qual apresenta um forte aspecto para o crescente avanço da produção agrícola (EMBRAPA, 2014).

Todavia, o que restringe o sucesso do setor da agricultura nesta região refere-se senão a ineficiência da preservação dos recursos hídricos e baixas recargas hídricas, causando grande impacto para o aumento da expansão agrícola. De acordo com informações da Agência Nacional de Águas (ANA) o ciclo da água tem ligação direta com clima, neste sentido as mudanças ocorridas no mesmo podem resultar em eventos climáticos extremos, comprometendo a oferta d'água para todos, o que exige medidas para aumentar a segurança hídrica e adaptações a novos cenários.

Dentre os municípios da região Nordeste, Bom Jesus da Lapa possui uma posição geográfica totalmente abrangida

\section{MATERIAIS E MÉTODOS}

O trabalho foi realizado no município de Bom Jesus da Lapa, sob coordenadas geográficas de $13^{\circ} 15^{\prime} 18^{\prime \prime}$ latitude sul e $43^{\circ} 25^{\prime} 05^{\prime \prime}$ de longitude oeste, pelo polígono das secas do estado da Bahia com estações secas no inverno e chuvas de verão, sem nenhum excedente hídrico, com precipitação anual de $833 \mathrm{~mm}$ (BEZERRA et al., 2010; INMET). No entanto, possui o principal afluente, o rio São Francisco, que percorre entorno de 70 quilômetros dentro do município (IBGE, 2017).

Por trata-se de espaços territoriais que absorve muito o impacto do clima semiárido, sendo propício à desertificação, o planejamento adequado dos recursos hídricos se torna a base para uma agricultura sustentável. Uma forma adequada para dimensionar a utilização da água é através do Balanço Hídrico Climatológico, no qual permite o conhecimento da disponibilidade hídrica no solo, além de permitir realizar a classificação climática da região (SANTOS et al., 2010).

Deste modo, é de suma importância conhecer e buscar estratégias, que direcione maneiras para o manejo de recursos hídricos. Diante disso, o presente trabalho tem o objetivo de analisar a disponibilidade hídrica através do balanço hídrico climatológico e realizar a classificação climática para o município de Bom Jesus da Lapa,

Bahia. em uma altitude média de $436 \mathrm{~m}$, na região do Médio São Francisco, estabelecido no oeste do estado da Bahia com o bioma classificado como Caatinga (Figura 1). Os 
tipos de solos dominantes da região são os Podzólicos Vermelho (IBGE, 2017).

Latossolos e Planossolos Solódicos,

Figura 1. Localização geográfica do município de Bom Jesus da Lapa, Bahia
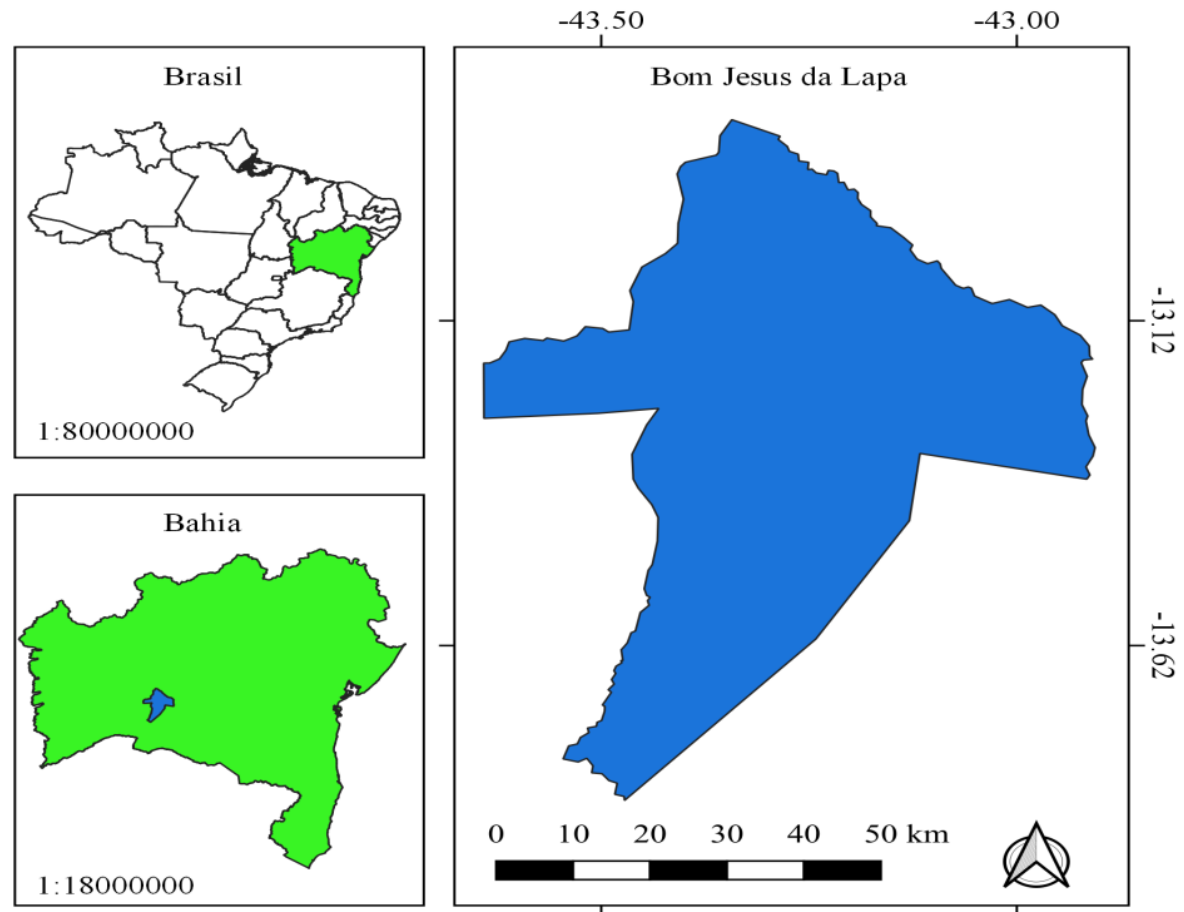

Para a realização do Balanço Hídrico Climatológico (BHC), foram utilizados dados mensais de precipitação $(\mathrm{mm})$ e temperatura $\left({ }^{\circ} \mathrm{C}\right)$, sendo esses dados provenientes da estação meteorológica convencional pertencente ao Instituto Nacional de Meteorologia (INMET), no período de 01 de janeiro de 1970 a dezembro de 2018, entretanto os anos de 1971, 1972-1981-1986, 1989 a 1991 não foram utilizados, pois não tinham dados disponíveis pela estação, com isso totalizou uma série histórica de 37 anos. Para o cálculo do BHC foi utilizado o método desenvolvido por Thorthwaite e Mather (1955), utilizando planilha elaborada por Rolim et al. (1998). Para a execução do BHC, foi adotado o valor de $100 \mathrm{~mm}$ para a capacidade de água disponível no solo (CAD).

A Classificação Climática foi estimada a partir do método de Thornthwaite e Mather (1955), que utiliza os dados de excesso e deficiência hídrica anual obtida do BHC para encontrar os índices: hídrico (Ih), aridez (Ia) e umidade (Iu), de acordo com as Equações 1, 2 e 3.

$$
\begin{aligned}
& \mathrm{Ih}=\left(\frac{\mathrm{EXC}}{\mathrm{ETP}}\right) \times 100 \quad \text { Equação } 1 \\
& \mathrm{Ia}=\left(\frac{\mathrm{DEF}}{\mathrm{ETP}}\right) \times 100 \quad \text { Equação } 2 \\
& \mathrm{Iu}=(\mathrm{Ih}-0,6) \times \mathrm{Ia} \quad \text { Equação } 3
\end{aligned}
$$

Em que:

$\mathrm{EXC}=$ excedente hídrico, em mm;

$\mathrm{ETP}=$ evapotranspiração total, em mm;

$\mathrm{DEF}=$ deficiência hídrica, em $\mathrm{mm}$;

Ih = índice hídrico;

Ia = índice de aridez;

$\mathrm{Iu}=$ índice de umidade.

A partir do cálculo de índice de umidade, foi consultada a chave para determinar o tipo climático do município (Tabela 1). Em seguida, com os índices de aridez e hídrico determinam-se os subtipos climáticos (Tabela 2). Também foi 
classificado o clima quanto ao índice térmico, que são definidos com base na evapotranspiração potencial anual, e os subtipos que dependem da relação porcentual ETP no verão/ETP anual (Tabela 3 e

Tabela 1. Chave inicial da classificação climática segundo Thornthwaite, baseado nos índices de umidade

\begin{tabular}{cc}
\hline Tipos Climáticos & Índice de Umidade (Iu) \\
\hline A -Super-úmido & $100 \leq \mathrm{Iu}$ \\
B4 - Úmido & $80 \leq \mathrm{Iu}<100$ \\
B3 - Úmido & $60 \leq \mathrm{Iu}<80$ \\
B2 - Úmido & $40 \leq \mathrm{Iu}<60$ \\
B1 - Úmido & $20 \leq \mathrm{Iu}<40$ \\
C2 - Sub-úmido & $00 \leq \mathrm{Iu}<20$ \\
C1 - Sub-úmido seco & $-33,33 \leq \mathrm{Iu}<00$ \\
D - Semi-árido & $-66,7 \leq \mathrm{Iu}<-33,33$ \\
E - Árido & $-100 \leq \mathrm{Iu}<-66,7$ \\
\hline
\end{tabular}

Fonte: Pereira et al. (2007).

Tabela 2. Segundo chave- Subtipos climáticos, segundo Thornthwaite, baseados nos índices de aridez e umidade

\begin{tabular}{|c|c|c|c|}
\hline $\begin{array}{c}\text { Climas úmidos } \\
\text { (A, B4, B3, B2, B1 e } \\
\text { C2) }\end{array}$ & $\begin{array}{l}\text { Índice de Aridez } \\
\text { (Ia) }\end{array}$ & $\begin{array}{l}\text { Climas secos } \\
\text { (C1, D e E) }\end{array}$ & Índice de umidade (Iu) \\
\hline $\begin{array}{l}\mathrm{r} \text { - pequena ou nenhuma } \\
\text { deficiência de água }\end{array}$ & $0-16,7$ & $\begin{array}{l}\mathrm{d}-\text { pequeno ou nenhum } \\
\text { excesso de água }\end{array}$ & $0-10$ \\
\hline $\begin{array}{c}\mathrm{s} \text { - moderada } \\
\text { deficiência } \\
\text { no verão }\end{array}$ & $16,7-33,33$ & $\begin{array}{c}\mathrm{s}-\text { moderado excesso } \\
\text { de inverno }\end{array}$ & $10-20$ \\
\hline $\begin{array}{l}\text { w - moderada } \\
\text { deficiência } \\
\text { no inverno }\end{array}$ & $16,7-33,33$ & $\begin{array}{c}\text { w - moderado excesso } \\
\text { de verão }\end{array}$ & $10-20$ \\
\hline $\begin{array}{c}\text { s2 - grande deficiência } \\
\text { no verão }\end{array}$ & $>33,33$ & $\begin{array}{c}\text { s2 - largo excesso } \\
\text { de inverno }\end{array}$ & 20 \\
\hline $\begin{array}{c}\text { w2 - grande deficiência } \\
\text { no inverno }\end{array}$ & $>33,33$ & $\begin{array}{c}\text { w2 - largo excesso } \\
\text { de verão }\end{array}$ & 20 \\
\hline
\end{tabular}

Fonte: Pereira. (2007).

Tabela 3. Terceira chave de classificação climática segundo Thornthwaite, baseado no índice térmico (ETo anual)

\begin{tabular}{cc}
\hline Tipo climático & Índice térmico (ETo anual) \\
\hline A' - megatérmico & $\geq 1.140$ \\
B'4 - mesotérmico & $997-1.140$ \\
B'3 - mesotérmico & $855-997$ \\
B'2 - mesotérmico & $712-855$ \\
B'1 - mesotérmico & $570-712$ \\
C'2 - microtérmico & $427-570$ \\
C'1 - microtérmico & $285-427$ \\
D' - tundra & $142-285$ \\
E' - gelo perpétuo & $<142$ \\
\hline
\end{tabular}

Fonte: Pereira et al. (2007). 
Tabela 4. Quarta chave de classificação climática segundo Thornthwaite, baseado na relação entre a ETP de verão (ETPv) e anual (ETP)

\begin{tabular}{cc}
\hline Subtipo climático & Concentração da ETP no verão (\%) \\
\hline a' & $<48 \%$ \\
b'4 & $48-51,9$ \\
b'3 & $51,9-5673$ \\
b'2 & $56,3-61,6$ \\
b'1 & $61,6-68,0$ \\
c'2 & $68,0-76,3$ \\
c'1 & $76,3-88,00$ \\
d' & $>88,00$ \\
\hline
\end{tabular}

Fonte: Pereira et al. (2007).

\section{RESULTADOS E DISCUSSÃO}

É possível observar na Tabela 5 no município em estudo, que sua temperatura média anual foi de $26,37^{\circ} \mathrm{C}$ e a precipitação média anual é de 792,66 mm. As menores temperaturas médias observadas foram encontradas nos meses de Junho e Julho, com 25,62 e $24,34{ }^{\circ} \mathrm{C}$ respectivamente, sendo que em para o mês de Julho verifica- se a menor precipitação $\left(0,25 \mathrm{~mm}\right.$ mês $\left.^{-1}\right)$, ETP $\left(96,43 \mathrm{~mm}\right.$ mês $\left.^{-1}\right)$ e ETR $(0,59 \mathrm{~mm}$ mês $\left.{ }^{-1}\right)$. Em contrapartida, a maior temperatura média de $28,34{ }^{\circ} \mathrm{C}$ foi verificada no mês de Outubro, que coincide com a máxima ETP 158,90 mm mês ${ }^{-1}$.

Tabela 5. Extrato das variáveis do BHC pelo método de Thornthwaite; Mather (1955), para o município de Bom Jesus da Lapa, no período de 1970 a 2018.

\begin{tabular}{ccccccccccc}
\hline Meses & $\mathbf{T}$ & $\mathbf{P}$ & $\mathbf{E T P}$ & $\mathbf{P - E T P}$ & $\begin{array}{c}\text { NEG- } \\
\mathbf{A C}\end{array}$ & $\begin{array}{c}\text { ARM } \\
\mathrm{mm}\end{array}$ & $\begin{array}{c}\text { ALT } \\
\mathrm{mm}\end{array}$ & $\begin{array}{c}\text { ETR } \\
\mathrm{mm}\end{array}$ & $\begin{array}{c}\text { DEF } \\
\mathrm{mm}\end{array}$ & $\begin{array}{c}\text { EXC } \\
\mathrm{mm}\end{array}$ \\
\hline Jan & 26,70 & 124,01 & 152,57 & $-28,56$ & $-163,64$ & 19,47 & $-6,44$ & 130,44 & 22,13 & 0,00 \\
Fev & 26,81 & 104,12 & 136,64 & $-32,51$ & $-196,16$ & 14,06 & $-5,40$ & 109,52 & 27,11 & 0,00 \\
Mar & 26,83 & 99,62 & 146,71 & $-47,10$ & $-243,25$ & 8,78 & $-5,28$ & 104,90 & 41,81 & 0,00 \\
Abr & 26,54 & 58,57 & 134,68 & $-76,11$ & $-319,37$ & 4,10 & $-4,68$ & 63,25 & 71,43 & 0,00 \\
Mai & 25,80 & 13,47 & 120,52 & $-107,05$ & $-426,41$ & 1,41 & $-2,70$ & 16,17 & 104,3 & 0,00 \\
Jun & 24,62 & 2,39 & 96,44 & $-94,05$ & $-520,47$ & 0,55 & $-0,86$ & 3,24 & 93,19 & 0,00 \\
Jul & 24,34 & 0,25 & 96,43 & $-96,18$ & $-616,65$ & 0,21 & $-0,34$ & 0,59 & 95,84 & 0,00 \\
Ago & 25,43 & 1,05 & 115,25 & $-114,20$ & $-730,85$ & 0,07 & $-0,14$ & 1,19 & 114,0 & 0,00 \\
Set & 27,32 & 11,67 & 143,99 & $-132,32$ & $-863,16$ & 0,02 & $-0,05$ & 11,72 & 132,2 & 0,00 \\
Out & 28,34 & 59,92 & 158,90 & $-98,98$ & $-962,14$ & 0,01 & $-0,01$ & 59,93 & 98,96 & 0,00 \\
Nov & 27,13 & 138,89 & 151,83 & $-12,93$ & $-975,07$ & 0,01 & 0,00 & 138,90 & 12,93 & 0,00 \\
Dez & 26,61 & 178,70 & 152,81 & 25,90 & $-135,08$ & 25,90 & 25,90 & 152,81 & 0,00 & 0,00 \\
\hline Totais & & 792,66 & 1606,76 & $-814,09$ & - & 74,58 & 0,00 & 792,66 & 814,0 & 0,00 \\
\hline Média & 26,37 & 66,06 & 133,90 & $-67,84$ & - & 129,69 & 6,44 & 66,06 & 67,84 & 0,00 \\
\hline
\end{tabular}

T: Temperatura média do ar; P: Precipitação; ETP: Evapotranspiração Potencial; NG-A: Negativo Acumulado; ARM: Armazenamento de água no solo; ALT: Alteração do armazenamento de água no solo; ETR: Evapotranspiração real; DEF: Deficiência Hídrica e EXC: Excedente Hídrico.

De uma maneira geral, é possível observar que a ETP foi maior nos períodos mais quentes do ano, ou seja, durante o verão. Isso se deve por que nesse período há maior quantidade de energia solar para condicionar a evapotranspiração, além de que os dias também são mais longos, aumentando assim a ETP (CAMARGO \& CAMARGO,2000). 
$\mathrm{Na}$ Figura 2 verifica-se a distribuição da ETP e ETR, sendo possível observar que a ETP foi maior para todos os meses, com exceção a Dezembro que possui valores iguais de ETP e ETR, com $152,81 \mathrm{~mm} \mathrm{mês}^{-1}$. Isso ocorreu porque neste mês houve a maior precipitação no município $\left(178,70 \mathrm{~mm}\right.$ mês $\left.^{-1}\right)$, o que consequentemente aumentou a quantidade de água disponibilizada no solo. De maneira oposta, o mês de Julho conta com a menor ETP e também menor ETR, o que se justifica pela menor precipitação ocorrida neste período e consequentemente, baixa umidade do solo.

De acordo com o BHC da série histórica analisada (Figura 3), o município
Bom Jesus da Lapa apresenta deficiência hídrica em 11 dos 12 meses do ano, valor esse que somado representa uma deficiência de $814,09 \mathrm{~mm}$, ou seja, mais que o dobro da precipitação média anual da região. Isso faz com que haja grandes problemas em culturas manejadas sem irrigação na região. Neste contexto, a instalação do Perímetro Irrigado Formoso na região pode contribuir significativamente, sendo que o setor de fruticultura irrigada representa $88 \%$ de toda área plantada e tem como principais

Figura 2. Variação dos dados mensais meteorológicos de precipitação e estimativas da evapotranspiração real e evapotranspiração de referência, em milímetros, para cada mês, no município de Bom Jesus da Lapa - BA (1970 a 2018).

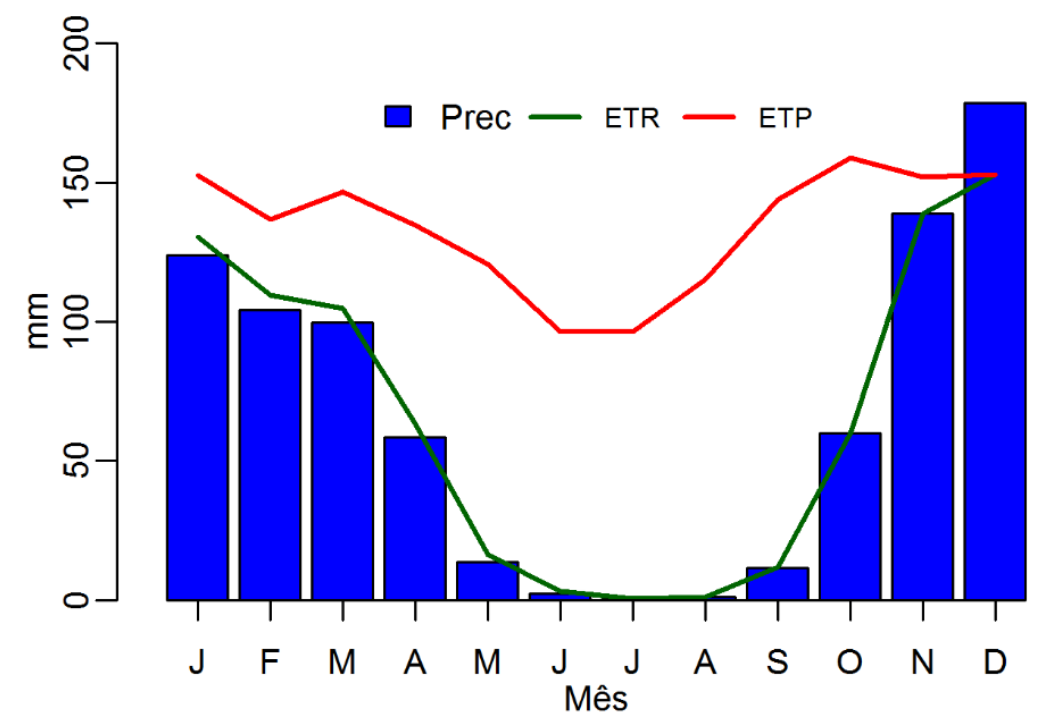

culturas a banana (85\%), seguida do mamão, melancia e coco (CODEVASF, 2013).

Os meses de Novembro e Dezembro apresentam as maiores chuvas (Figura 2), e isso fez com que houvesse uma redução da deficiência hídrica da região, passando de
98,96 mm no mês de Outubro para 12,93 mm em Novembro. No mês de Dezembro já é possível verificar uma leve reposição de $25,90 \mathrm{~mm} \mathrm{mês}^{-1}$, o que se deve as elevadas chuvas ocorridas nesse mês $(178,70 \mathrm{~mm})$, superando assim a ETP. 
Figura 3: Balanço hídrico climatológico, em milímetros, de todos os meses do ano, para o município de Bom Jesus da Lapa - BA

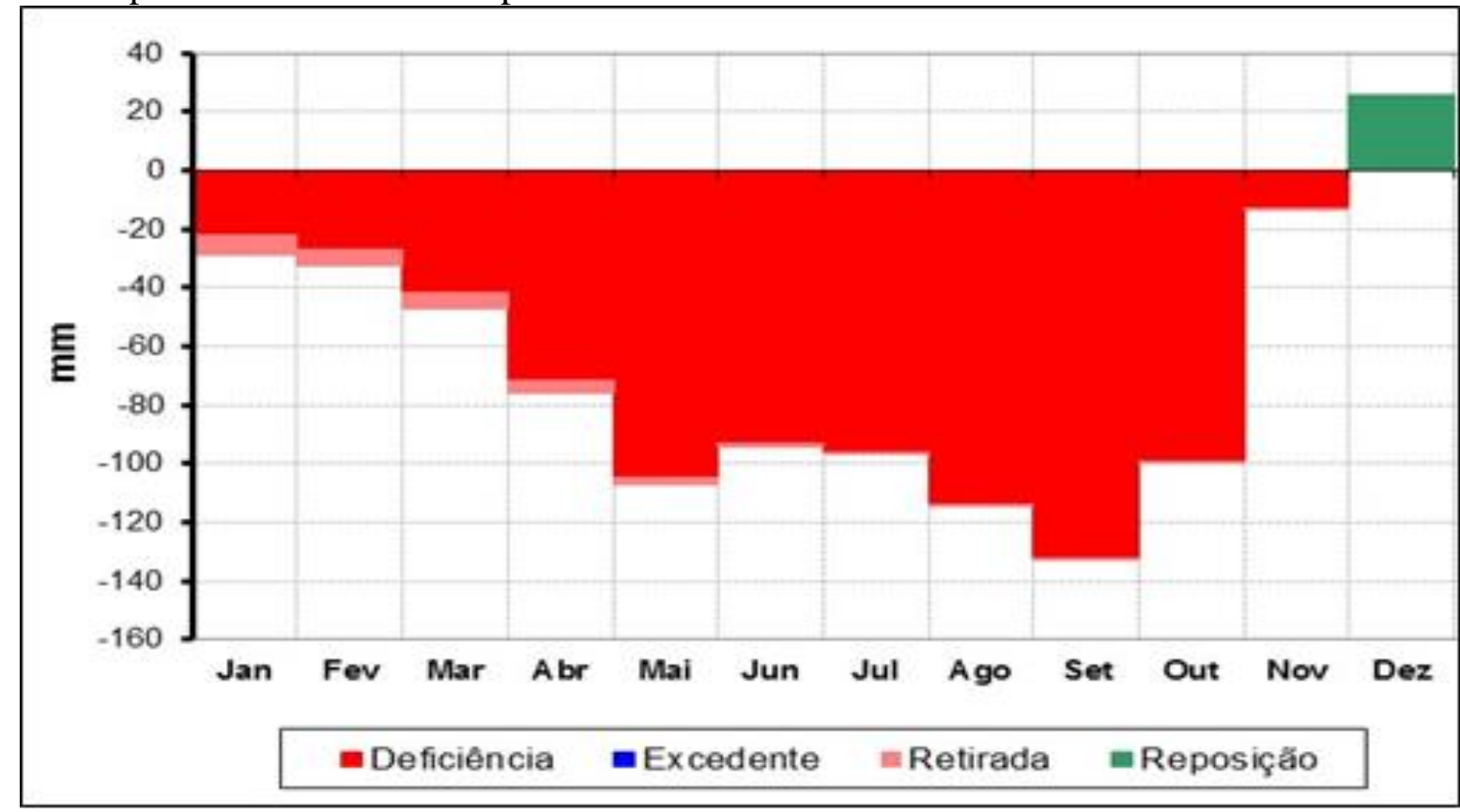

A partir dos índices do BHC, foi possível realizar a classificação climática (Tabela 2). Por meio da primeira chave, o valor do índice de umidade (Iu) foi $-30,40$, correspondendo a tipologia $\mathrm{C}_{1}$ que indica clima sub-úmido seco. Em seguida, foi verificado subdivisão do tipo climático por meio da relação Ia/Ih $(50,67)$ encontrando a letra $\mathrm{w}_{2}$ que representa grande deficiência hídrica no inverno.

Posteriormente foi analisada a terceira chave que caracterizou a subdivisão do tipo climático com base na eficiência térmica pelo total da evapotranspiração potencial anual, onde foi determinada como megatérmico pela letra $A^{\prime}$. Por último, determinou-se a evapotranspiração potencial no verão em relação à evapotranspiração anual, sendo o subtipo a'. Com isso, a classificação climática obtida apresentou os caracteres $\mathrm{C}_{1} \mathrm{w}_{2} \mathrm{~A}^{\prime}$ a' que corresponde ao clima sub-úmido seco com grande deficiência hídrica no inverno, megatérmico com concentração de evapotranspiração potencial no verão igual a $36,64 \%$ em relação à evapotranspiração anual.

Tabela 6. Classificação climática do Município de Bom Jesus da Lapa, BA. Método de Thornthwaite (1948)

\begin{tabular}{ccccc}
\hline Ih $(\%)$ & Ia (\%) & Iu (\%) & ETP anual & ETPv/ETPa (\%) \\
\hline 0,00 & 50,67 & $-30,40$ & $1.606,76$ & 36,64 \\
- & $\mathrm{w}_{2}$ & $\mathrm{C}_{1}$ & $\mathrm{~A}^{\prime}$ & a'
\end{tabular}

Índices: hídrico (Ih), de aridez (Ia) e de umidade (Iu), relação entre o índice de aridez e hídrico, relação entre a evapotranspiração potencial no verão (ETPv) e a evapotranspiração potencial (ETP) total anual.

No município de Bom Jesus da Lapa, o estudo da classificação climática torna-se muito importante devido ser uma metodologia simples de fácil entendimento podendo contribuir para a agricultura local. Segundo Andrade Júnior et al., (2005) tal classificação tem por finalidade 0 conhecimento de uma determinada área ou 
região e entendimento do clima, além de informações biogeográficas, o que

possibilita obtenção de informações relevantes do potencial agrícola por exemplo. Nesta vertente, informações precisas e detalhadas da classificação

\section{CONCLUSÕES}

Os meses de Janeiro a Novembro compreendem os meses de deficiência hídrica, enquanto que Dezembro é representado por reposição hídrica, cuja precipitação foi $178,70 \mathrm{~mm}^{\text {mês }}{ }^{-1}$, e a

A diferença entre a precipitação e a evapotranspiração potencial (P-ETP) foi de $25,90 \mathrm{~mm}$.

\section{REFERÊNCIAS BIBLIOGRÁFICAS}

ANA- AGÊNCIA NACIONAL DE ÁGUAS, 2019. Disponível em: <https://www.ana.gov.br/acesso-ainformacao/institucional/sobre-a-ana $>$. Acesso em:18 de Setembro de 2019. ANDRADE JÚNIOR, A. S.; BASTOS, E. A.; BARROS, A. H. C.; SILVA, C. O.; GOMES, A. A. N. Classificação climática e regionalização do semiárido do Estado do Piauí sob cenários pluviométricos distintos, Revista Ciência Agronômica, v. 36, n. 2, p. $143-151,2005$.

BEZERRA, J. R. C.; AZEVEDO, P. V.; SILVA, B. B.; DIAS, J. M. Evapotranspiração e coeficiente de cultivo do algodoeiro BRS-200 Marrom, irrigado.

Revista Brasileira de Engenharia Agrícola e Ambiental, Campina Grande, v. 14, n. 6, p. 625-632, 2010.

CAMARGO, M. B. P.; CAMARGO, A. P. Uma Revisão Analítica da Evapotranspiração Potencial. Bragantia, Campinas, v.59, n.2, p. 125-137, 2000.

CODEVASF - Companhia de

Desenvolvimento dos Vales do São

Francisco e do Parnaíba.

EMBRAPA, Empresa Brasileira de Pesquisa Agropecuária. Solos do climática para o município de Bom Jesus da Lapa é de grande valia para os produtores, auxiliando no processo de planejamento produtivo e reduzindo os riscos na produção.

Pela classificação climática, o município ficou descrito como $\mathrm{C}_{1} \mathrm{w}_{2} \mathrm{~A}^{\prime} \mathrm{a}^{\prime}$ representando um clima megatérmico subúmido seco com grande deficiência hídrica no inverno.

Nordeste. Unidade de execução de Pesquisa e Desenvolvimento de Recife, 2014, p. 8. Disponível em: $<$ https://www.embrapa.br/busca-depublicacoes/-/publicacao/1003864/solosdo-nordeste> Acesso em:18 de Setembro de 2019.

IBGE- INSTITUTO BRASILEIRO DE GEOGRAFIA E ESTATÍSTICA (2017). Cidades. Bom Jesus da Lapa- BA. Disponível em < https://cidades.ibge.gov.br/brasil/ba/bomjesus-da-lapa/historico> Acesso em: 19 mar. 2019.

INMET. Instituto Nacional de Meteorologia. Clima [Internet]. Disponível em: http://www.inmet.gov.br/portal/. Acesso em: 25 abr. 2019.

JESUS, J. B. Estimativa do balanço hídrico climatológico e classificação climática pelo método de Thornthwaite e Mather para o município de Aracaju-SE. Scientia Plena, Sergipe, v. 11, n. 5. 2015.

OLIVEIRA, ELIEGE A. DE P.; ZUCARELI, C.; PRETE, C. E.; ZAMUNER, D. Potencial osmótico do substrato na germinação de sementes e desenvolvimento inicial de plântulas de milho doce. Revista Brasileira de 
Ciências Agrárias, v. 9, n. 4, p. 477-482, 2014.

PEREIRA, A. R.; ANGELOCCI, L. R.; SENTELHAS, P. C. Meteorologia Agrícola. Piracicaba-SP, 2007.

ROLIM, G. S.; SENTELHAS, P. C.; BARBIERI, V. Planilhas no ambiente EXCEL para os cálculos de balanços hídricos: normal, sequencial, de cultura e de produtividade real e potencial. Revista Brasileira de Agrometeorologia, v.6, p.133-137, 1998.

SALES, R. A.; OLIVEIRA, E. C.; LIMA, M. J. A.; GELCER, E. M., SANTOS. R. A.; LIMA, C. F. Ajuste dos coeficientes das equações de estimativa da evapotranspiração de referência para São Mateus, ES. Revista Irriga, Botucatu, v. 23, n. 1, p. 154-167, 2018.

SANTOS, G. O.; HERNANDEZ, F. B. T.; ROSSETTI, J. C. Balanço hídrico como ferramenta ao planejamento agropecuário para a região de Marinópolis, Noroeste do Estado de São Paulo. Revista Brasileira de Agricultura Irrigada, v.4, $\mathrm{n}^{\circ} .3,2010$. THORNTHWAITE, C. W.; MATHER, J. $\mathrm{R}$. The water balance. Publications in climatology. Laboratory of Climatology, New Gersey, v.8, p. 104, 1955. 